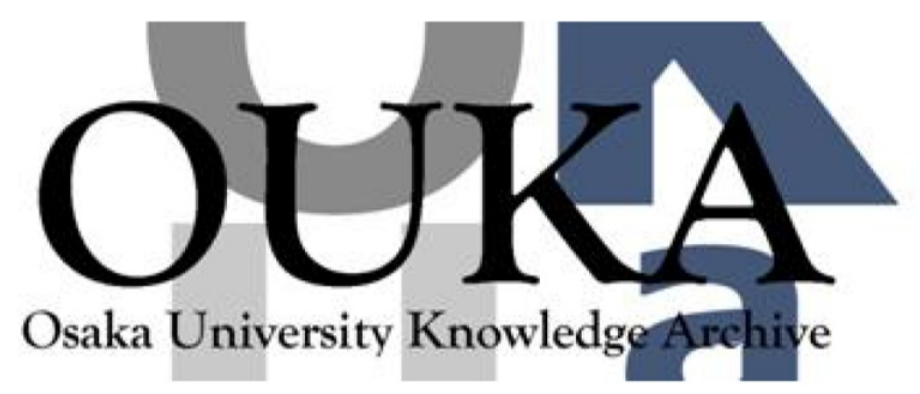

\begin{tabular}{|c|c|}
\hline Title & $\begin{array}{l}\text { Selective labeling of a single organelle by } \\
\text { using two-photon conversion of a } \\
\text { photoconvertible fluorescent protein }\end{array}$ \\
\hline Author (s) & $\begin{array}{l}\text { Watanabe, Wataru; Shimada, Tomoko; Matsunaga, } \\
\text { Sachihiro et al. }\end{array}$ \\
\hline Citation & $\begin{array}{l}\text { Proceedings of SPIE - The International Society } \\
\text { for Optical Engineering. } 6860 \text { p. } 68601 \mathrm{~B}\end{array}$ \\
\hline Issue Date & $2008-02-15$ \\
\hline oaire:version & VoR \\
\hline URL & https://hdl. handle. net/11094/79147 \\
\hline rights & $\begin{array}{l}\text { Copyright } 2008 \text { Society of Photo Optical } \\
\text { Instrumentation Engineers (SPIE). One print or } \\
\text { electronic copy may be made for personal use } \\
\text { only. Systematic reproduction and distribution, } \\
\text { duplication of any material in this publication } \\
\text { for a fee or for commercial purposes, or } \\
\text { modification of the contents of the publication } \\
\text { are prohibited. }\end{array}$ \\
\hline Note & \\
\hline
\end{tabular}

Osaka University Knowledge Archive : OUKA

https://ir. Library. osaka-u. ac. jp/

Osaka University 


\section{Selective labeling of a single organelle by using two-photon conversion of a photoconvertible fluorescent protein}

Watanabe, Wataru, Shimada, Tomoko, Matsunaga, Sachihiro, Kurihara, Daisuke, Arimura, Shin-ichi, et al.

Wataru Watanabe, Tomoko Shimada, Sachihiro Matsunaga, Daisuke Kurihara, Shin-ichi Arimura, Nobuhiro Tsutsumi, Kiichi Fukui, Kazuyoshi Itoh, "Selective labeling of a single organelle by using two-photon conversion of a photoconvertible fluorescent protein," Proc. SPIE 6860, Multiphoton Microscopy in the Biomedical Sciences VIII, 68601B (15 February 2008); doi: $10.1117 / 12.768745$ 


\title{
Selective labeling of a single organelle by using two-photon conversion of a photoconvertible fluorescent protein
}

\author{
Wataru Watanabe ${ }^{a^{*}}$, Tomoko Shimada ${ }^{b}$, Sachihiro Matsunaga ${ }^{c}$, Daisuke Kurihara ${ }^{c}$, Shin-ichi \\ Arimura $^{d}$, Nobuhiro Tsutsumi ${ }^{d}$, Kiichi Fukui ${ }^{c}$, and Kazuyoshi Itoh ${ }^{b}$ \\ ${ }^{a}$ Photonics Research Institute, National Institute of Advanced Industrial Science and Technology \\ (AIST), 1-8-31, Midorigaoka, Ikeda, Osaka 563-8577, Japan \\ ${ }^{\mathrm{b}}$ Department of Material and Life Science, Graduate School of Engineering, Osaka University, \\ 2-1, Yamadaoka, Suita, Osaka 565-0871, Japan \\ ${ }^{c}$ Department of Biotechnology, Graduate School of Engineering, Osaka University \\ 2-1, Yamadaoka, Suita, Osaka 565-0871, Japan \\ ${ }^{d}$ Graduate School of Agricultural and Life Sciences, University of Tokyo \\ 1-1-1 Yayoi, Bunkyo-ku, Tokyo 113-8657, Japan
}

\begin{abstract}
We present space-selective labeling of organelles by using two-photon conversion of a photoconvertible fluorescent protein with near-infrared femtosecond laser pulses. Two-photon excitation of photoconvertible fluorescent-protein, Kaede, enables space-selective labeling of organelles. We alter the fluorescence of target mitochondria in a tobacco BY2 cell from green to red by focusing femtosecond laser pulses with a wavelength of $750 \mathrm{~nm}$.
\end{abstract}

Keywords: femtosecond laser, photoactive protein, photo-convertible protein, organelles, two-photon excitation

\section{INTRODUCTION}

Near-infrared femtosecond lasers have recently attracted much attention in cell biology. Femtosecond lasers can be used to image subcellular structures using multi-photon excitation microscopy without compromising viability $[1,2]$. Femtosecond lasers operating in the near infrared region have attractive advantages compared with conventional ultraviolet lasers, including high resolution, a background-free signal, low background scattering, deep penetration into thick samples, and reduced photon-induced damage. This, a two-photon microscope equipped with a femtosecond laser has become a powerful tool for investigating biological phenomena [1-3] and has been applied to fluorescence recovery after photobleaching (FRAP) [4], and nanosurgery [5-7] in vivo.

Photoactivation and photoconversion are increasingly recognized as powerful methods for studying the dynamic processes in living cells [8-21]. Photoactivation and photoconversion enable selective conversion or activation of fluorescence signals after optical illumination, and are recognized as powerful tools for studying the dynamic processes of fluorescently labeled individual cells, organelles, and proteins.

Photoactivation refers to the transformation of an essentially nonfluorescent molecule into a highly fluorescent product by the absorption of light [8-12]. Photoactivatable green fluorescent protein (PAGFP), one of these designed proteins, shifts its excitation peak from 400 to $504 \mathrm{~nm}$ upon irradiation at $410 \mathrm{~nm}$ [9]. Transport processes, such as diffusion and flow, are studied by confining the activation of PA-GFP to a region of interest and by observing the changes in the fluorescence associated with activated proteins entering or leaving the volume.

Photoconversion describes the conversion of a fluorescent molecule into one with altered fluorescence properties by

*wataru.watanabe@aist.go.jp; phone 8172751 8984; fax 81727514027

Multiphoton Microscopy in the Biomedical Sciences VIII, edited by Ammasi Periasamy, Peter T. C. So, Proc. of SPIE Vol. 6860, 68601B, (2008) · 1605-7422/08/\$18 - doi: 10.1117/12.768745 
the absorption of light [13-21]. Rational design and random mutagenesis have produced fluorescent proteins with photoactive properties suitable for cellular applications. For example, Kaede, a cloned fluorescent protein extracted from the open brain coral Trachyphyllia geoffroyi, has an emission colour that can be irreversibly changed from green to red by exposure to ultraviolet light [18-21].

In order to make photoactivation a viable tool for intracellular applications, it is important to selectively activate Photoactivate protein or convert Photoconvertible protein within a very small region, such as inside an organelle, or a cellular compartment. Florescent protein can be excited by two-photon excitation in volumes of $<1$ femto-liter by focusing near-infrared femtosecond laser pulses and would be ideal for targeted photoactivation or photoconversion within cells. It was recently reported that PA-GFP is activated via a two-photon excitation process [10]. A study of the two-photon activation process of PA-GFP is also available [12]. Two-photon activation of PA-GFP provides a unique tool for probing cellular transport processes, because activation is strictly limited to the subfemtoliter optical volume of the two-photon volume. Two-photon conversion of Kaede has been conducted; however, previous studies on photoactivation or photoconversion using near-infrared femtosecond laser pulses suffered from the problem of low spatial resolution.

In this paper, we report on selective labeling of target organelles by using two-photon conversion of a photoconvertible fluorescent protein with near-infrared femtosecond laser pulses. Photoconversion of Kaede was performed using near-infrared femtosecond laser pulses with a wavelength of $750 \mathrm{~nm}$. Femtosecond laser pulses were focused into a target mitochondrion to alter the fluorescence from green to red. We performed selective labeling of mitochondria in a living tobacco BY-2 cell using two-photon photoconversion of Kaede.

\section{TWO-PHOTON CONVERSION}

Figure 1 shows schematic for photoconversion of an intracellular object by focusing near-infrared femtosecond laser pulses. In this study, we demonstrated selective labeling of organelles by using two-photon conversion of Kaede, and tracking the dynamics a single mitochondrion. The ability to label selective organelles is a potentially promising method for analysis of intracellular structures by tracking the dynamics of a single organelle in a living cell, revealing the mechanisms involved in organelle dynamics.

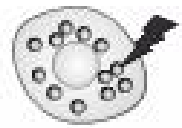

Femtosecond laser irradiation

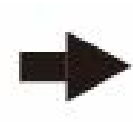

\section{Photoconversion of an intracellular object}

Figure 1: Schematic for photoconversion of an intracellular object by focusing near-infrared femtosecond laser pulses.

\section{EXPERIMENTAL SETUP}

In the experiments, we used fixed BY-2 cells whose mitochondria were labeled with Kaede [22]. Figure 2 shows a schematic diagram of the setup used for labeling organelle within a cell using a femtosecond Ti:sapphire laser oscillator. The laser scanning microscope was adapted from an Olympus FV300 scanning unit combined with an Olympus IX71 inverted microscope. A collimated $\mathrm{CW}$ laser beam of a He-Ne laser (wavelength $543 \mathrm{~nm}$ ) or an Ar-ion laser (wavelength $488 \mathrm{~nm}$ ) was reflected by dichroic mirrors DM1 and DM2 and then focused into the cells through an oilimmersion objective lens (OB; Olympus Corporation, PlanApo60×Oil, NA 1.4). The back-propagated one-photon fluorescence was collected using the same objective lens and detected with photomultiplier tubes (PMT1 and PMT2; Hamamatsu Photonics, R928P). Bandpass filters BP1 (transmission wavelength: $510 \mathrm{~nm}-540 \mathrm{~nm}$ ) and BP2 (transmission wavelength: $560 \mathrm{~nm}-600 \mathrm{~nm}$ ) were placed before PMT1 and PMT2. Two-dimensional confocal crosssectional $x y$ images were obtained by scanning the focused laser beams in the cross-sectional plane with a pair of highspeed galvanometer mirrors (GM; Cambridge, 6210) inside the laser-scanning microscope. Scanning in the depth direction was achieved by moving the objective lens with a stepping motor to obtain three-dimensional confocal images. 
Irradiation with femtosecond laser pulses was performed using a mode-locked Ti:sapphire laser oscillator with a wavelength of $750 \mathrm{~nm}$ and a repetition rate of $76 \mathrm{MHz}$ (Coherent, Mira). The laser pulses passed through a Faraday isolator (FI) to block reflections from the optical components. The laser pulses then passed through a series of SF10 prisms (P1, P2) to compensate for the dispersion of the optical components in the light path and the microscope. To fill the aperture of the objective lens (OB), the beam was reduced to $2 \mathrm{~mm}$ in diameter with a pair of lenses (L1, L2), and it was then directed onto a pair of GM via DM1 and DM2. Femtosecond laser pulses were focused into the BY-2 cells through the oil-immersion objective. The number of pulses supplied was selected by an electro-magnetic shutter (Sigma Koki, $\Sigma-65 \mathrm{~L})$.

The following protocol is used for the intracellular photoconversion experiment. We select a cell and record its green fluorescence with one-photon excitation at $488 \mathrm{~nm}$. In order to compare red fluorescence before and after femtosecond laser irradiation, one-photon fluorescence images of the BY-2 cells were obtained by excitation with CW He-Ne laser at $543 \mathrm{~nm}$. Next, we set the focal point of the objective lens to a target organelle inside the BY-2 cell. By opening the shutter, femtosecond laser pulses were focused into the target. Photoconversion of Kaede was performed around the focal volume of femtosecond laser pulses. After femtosecond laser irradiation, one-photon red and green fluorescence images of the HeLa cells were obtained by excitation with the cw He-Ne laser or $\mathrm{Ar}^{+}$laser.

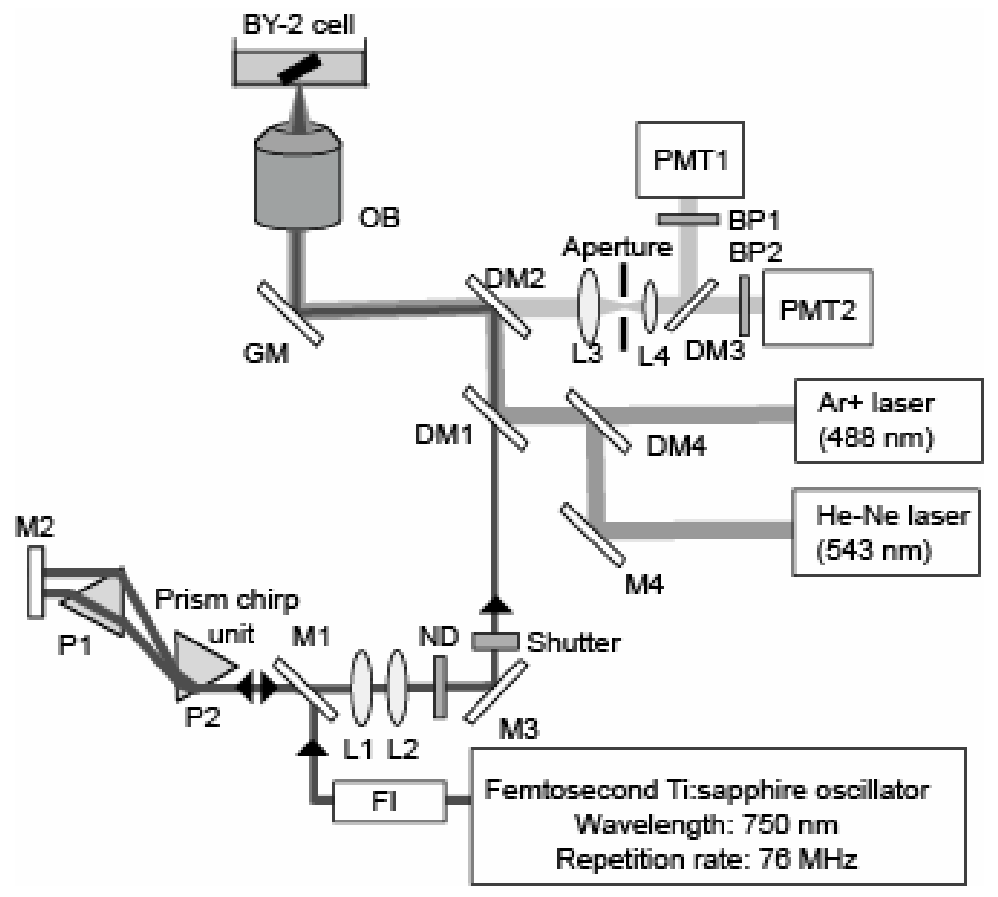

Figure 2: Schematic diagram of the experimental setup. FI, Faraday isolator; P, SF10 prism; M mirror; L, lens; ND, neutral density filter; DM, dichroic mirror; OB, objective lens; GM, pair of galvanometer mirrors; PMT, photomultiplier tube.

\section{EXPERIMENTAL RESULTS}

Figure 3 shows stacked 3D confocal fluorescence images before and after femtosecond laser irradiation. Green fluorescence images were obtained through a bandpass filter (transmission wavelength: 510 to $540 \mathrm{~nm}$, left column of Fig. 3), and red fluorescence images were obtained through another bandpass filter (transmission wavelength: 560 to $600 \mathrm{~nm}$, right column of Fig. 3). Laser pulses with an energy of $0.026 \mathrm{~nJ} / \mathrm{pulse}(2 \mathrm{~mW})$ were focused at the mitochondrion indicated by the arrow. Local green-to-red conversion was achieved by femtosecond laser irradiation within region of interest. After photoconversion by the femtosecond laser irradiation, the red fluorescent signal appeared. The results demonstrate that the fluorescence from target mitochondria was converted from green to red. The 
results demonstrate that spatially selective labeling of a single organelle can be performed in 3D space. The results presented here demonstrate that labeling by two-photon conversion is a powerful tool for dynamic analysis of a single organelle. Introduction of a photoconvertible fluorescent label enabled precise photolabeling in a sub-femtoliter focal volume.

(A)

Before femtosecond

laser iradiation

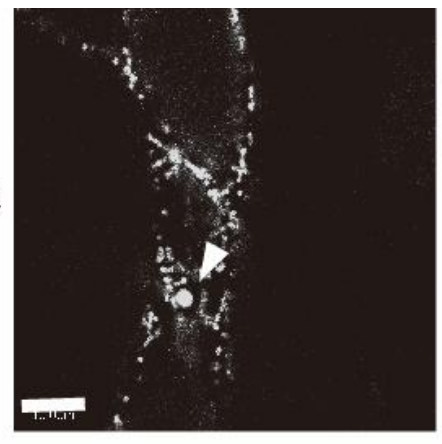

(B)

After femtosecond laser iradiation

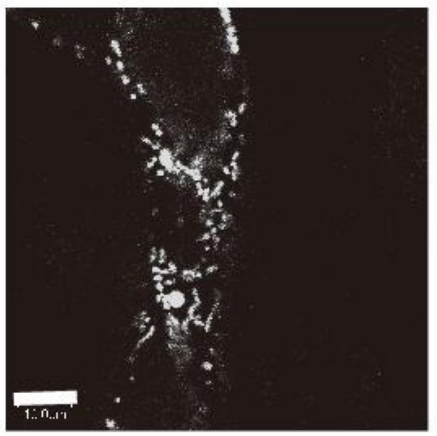

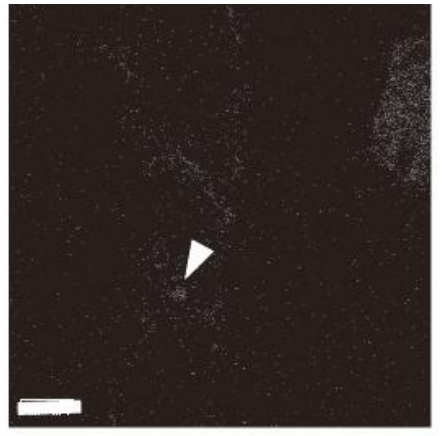

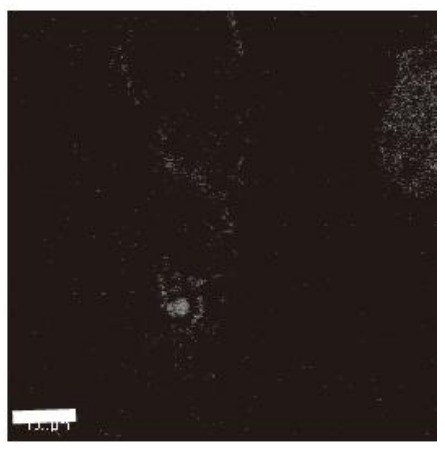

Figure 3: Selective photoconversion of mitochondria with Kaede in a fixed BY-2 cell. Left column, stacked 3D confocal images of green fluorescence obtained through bandpass filter (BP; 510 to $540 \mathrm{~nm}$ ); right column, stacked 3D confocal images of red fluorescence obtained through bandpass filter (560 to $600 \mathrm{~nm}$ ); right column, (A) before femtosecond laser pulse irradiation and (B) after femtosecond laser pulse irradiation. Mitochondria were photoconverted from green to red by $750-\mathrm{nm}$ femtosecond laser pulses with an energy of $0.026 \mathrm{~nJ} / \mathrm{pulse}$. A focal point is indicated by an arrow.

Scale bar: $10 \mu \mathrm{m}$.

Photoconversion using two-photon excitation offers advantages, including high resolution, deep penetration into thick samples, and reduced photon-induced damage. Photoconversion with near-infrared light is less harmful to cells than UV light. Two-photon excitation also allows selective photoconversion at any location in the cell within a photoconversion volume of a few femtoliters down to less than one femtoliter. 


\section{ACKNOWLEDGMENT}

A part of this work was supported by JSPS and DFG under the German Research Cooperative Program (W.W.). This work was supported by Grant-in-Aid for Scientific Research from the Ministry of Education, Science, Culture, Sports, Science and Technology of Japan, awarded to one of the authors (S.M.; grant no. 18687005).

\section{CONCLUSION}

We present femtosecond laser photoconversion of organelles using a Ti:sapphire laser oscillator The two-photon spot allows the selective activation at any location within the cell with subfemtoliter activation volumes. Photoconversion of mitochondria in a tobacco BY-2 cell with focused femtosecond laser pulses was demonstrated. Two-photon conversion of Kaede provides a powerful tool for probing movement processes in cells. This application demonstrates the attractive features of two-photon photoactivation over traditional techniques, such as photobleaching, for studying transport processes in cells.

\section{REFERENCES}

1. W. Denk, J. H. Strickler, and W. W. Webb, "Two-photon laser scanning fluorescence microscopy," Science 248, pp. 73-76, 1990.

2. K. König, "Multiphoton microscopy in life sciences," J. Microsc. 200, 83-104 (2000).

3. K. Isobe, W. Watanabe, S. Matsunaga, T. Higashi, K. Fukui, K.Itoh, "Multi-spectral two-photon excited fluorescence microscopy using supercontinuum light source,” Jpn. J. App. Phys. 44, L167-L169 (2005).

4. T. Higashi, S.Matsunaga, K.Isobe, A. Morimoto, T. Shimada, S. Kataoka, W. Watanabe, S. Uchiyama, K. Itoh, and K. Fukui, "Histone H2A mobility is regulated by its N- and C-terminal tails, and acetylation of core histone tails," Biochem. Biophys. Res.Commun 357, 627-632 (2007).

5. W. Watanabe, N. Arakawa, S. Matsunaga, T. Higashi, K. Fukui, K. Isobe, and K. Itoh, "Femtosecond laser disruption of subcellular organelles in a living cells," Opt. Exp. 12, 4203-4213 (2004).

6. W. Watanabe, S. Matsunaga, T. Shimada, T. Higashi, K. Fukui, and K. Itoh, "Femtosecond laser disruption of mitochondria in living cells," Med. Laser Appl., 20, 185-191 (2005).

7. T. Shimada, W. Watanabe, S. Matsunaga, T. Higashi, H. Ishii, K. Fukui, K. Isobe, and K. Itoh, "Intracellular disruption of mitochondria in a living HeLa cells with a 76-MHz femtosecond laser oscillator," Opt. Exp. 13 98699880 (2005).

8. J. Lippincott-Schwartz and G. H. Patterson, "Development and use of fluorescent protein markers in living cells," Science 300, 87-91 (2003).

9. G. H. Patterson and J. Lippincott-Schwartz, "A photoactivatable GFP for selective photolabeling of proteins and cells," Science 297, 1873-1877 (2002).

10. J. N. Post, K. A. Lidke, B. Rieger, and D. J. Arndt-Jovin, "One- and two-photon photoactivation of a paGFPfusion protein in live Drosophila embryos," FEBS Lett. 579, 325-330 (2005).

11. M. Karbowski, D. Arnoult, H. Chen, D. C. Chan, C. L. Smith, and R. J. Youle, "Quantitation of mitochondrial dynamics by photolabeling of individual organelles shows that mitochondrial fusion is blocked during the Bax activation phase of apoptosis," J. Cell Biol. 164, 493-499 (2004).

12. M. Schneider, S. Barozzi, I. Testa, M. Faretta and A. Diaspro, "Two-photon activation and excitation properties of PA-GFP in the 720-920 nm region," Biophys J. 89, 1346-1352 (2005).

13. D. M. Chudakov, V. V. Belousov, A. G. Zaraisky, V. V. Novoselov, D. B. Staroverov, D. B. Zorov, S. Lukyanov, and K. A. Lukyanov, "Kindling fluorescent proteins for precise in vivo photolabeling," Nature Biotechnol. 21, 191-194 (2003).

14. D. M. Chudakov, V. V. Verkhusha, D. B. Staroverov, E. A. Souslova, S. Lukyanov, and K. A. Lukyanov, "Photoswitchable cyan fluorescent protein for protein tracking," Nature Biotechnol. 22, 1435-1439 (2004).

15. Y. Chen, P. J. Macdonald, J. P. Skinner, G. H. Patterson, and J. D. Muller, "Probing nucleocytoplasmic transport by two-photon activation of PA-GFP," Microscopic Res. Tech. 69, 220-226 (2006).

16. J. Wiedenmann, S. Ivanchenko, F. Oswald, F. Schmitt, C. Rocker, A. Salih, K. D. Spindler, G. U. Nienhaus, "EosFP, a fluorescent marker protein with UV-inducible green-to-red fluorescence conversion," Proc Natl. Acad. Sci. USA 101, 15905-15910 (2004). 
17. N. G. Gurskaya, V. V. Verkhusha, A. S. Shcheglov, D. B. Staroverov, T. V. Chepurnykh, A. F. Fradkov, S. Lukyanov, and K. A Lukyanov, "Engineering of a monomeric green-to-red photoactivatable fluorescent protein induced by blue light," Nature Biotechnol. 24, 461-465 (2006).

18. R. Ando, H. Hama, M. Yamamoto-Hino, H. Mizuno and A. Miyawaki, "An optical marker based on the UVinduced green-to-red photoconversion of a fluorescent protein," Proc. Natl. Acad. Sci. USA 99, 12651-12656 (2002).

19. H. Mizuno, T. Kumar Mal, K. I. Tong, R. Ando, T. Furuta, M. Ikura, and A. Miyawaki, "Photo-induced peptide cleavage in the green-to-red conversion of a fluorescent protein," Mol. Cell 12, 795-1058 (2003).

20. H. Tsutsui, S. Karasawa, H. Shimizu, N. Nukina, and A. Miyawaki, "Semi-rational engineering of a coral fluorescent protein into an efficient highlighter," EMBO Rep. 6, 233-238 (2005).

21. S. Arimura, J. Yamamoto, G. P. Aida, M. Nakazono, and N. Tsutsumi, "Frequent fusion and fission of plant mitochondria with unequal nucleoid distribution," Proc. Natl. Acad. Sci. USA 101, 7805-7808 (2004).

22. W. Watanabe, T. Shimada, S. Matsunaga, D. Kurihara, S. Arimura, N. Tsutsumi, K. Fukui, K. Isobe, and K. Itoh, "Single organelle tracking by two-photon conversion," Opt. Express 15, 2490-2498 (2007) 